\title{
Étude de la propagation du plasma produit par ablation laser pour différentes cibles, dans le vide et dans différents gaz ambiants
}

\author{
C. Vivien, J. Hermann et C. Boulmer-Leborgne
}

Laboratoire GREMI, CNRS, Université d'Orléans, BP. 6759, 45067 Orléans cedex 2, France

\begin{abstract}
The purpose of this work is the study of the plasma induced by UV laser interaction with solid targets. A frequency quadrupled Nd:Yag laser beam $(266 \mathrm{~nm})$ of $5 \mathrm{~ns}$ pulse duration is focused to a power density in the $\mathrm{GW} \mathrm{cm}^{-2}$ range. Experiments are carried out on various target materials ( $\mathrm{Al}$, $\mathrm{Si}, \mathrm{Ti}, \mathrm{Cu}, \mathrm{Zr}, \mathrm{Hf}$ ) in vacuum and in low pressure atmospheres of several gases ( $\mathrm{He}, \mathrm{Ar}, \mathrm{Xe}, \mathrm{N}_{2}$ ). Optical emission spectroscopy and fast CCD (Charge Coupled-Device array) imaging in-situ measurements are performed to investigate the composition and the propagation of the plasma. The influence of the material/gas characteristics on the plasma propagation is studied.
\end{abstract}

L'étude présentée ici concerne l'expansion et la composition du panache plasma produit par interaction laser-matière dans les conditions expérimentales de dépôt dans le but de mieux comprendre les mécanismes fondamentaux mis en jeu. Des mesures résolues dans le temps par spectroscopie optique d'émission et par imagerie (caméra rapide CCD intensifiée) ont été effectuées.

Le laser utilisé est un Nd:Yag (Brillant Quantel) quadruplé en fréquence $(266 \mathrm{~nm})$ de curée d'impulsion $\tau=5 \mathrm{~ns}$. Le faisceau est focalisé sur différentes cibles ( $\mathrm{Al}, \mathrm{Si}, \mathrm{Ti}, \mathrm{Cu}, \mathrm{Zr}, \mathrm{Hf}$ ) (4 à $5 \mathrm{GWcm}^{-2}$ ). Les cibles sont vaporisées dans le vide ou dans un gaz ambiant à basse pression $\left(5.10^{-2}\right.$ à $1 \mathrm{mb}$ dans $\mathrm{He}, \mathrm{Ar}, \mathrm{Xe}$, ou $\left.\mathrm{N}_{2}\right)$. Les deux méthodes d'analyse utilisées sont complémentaires : les photos permettent de suivre la propagation $2 \mathrm{D}$ du plasma et la spectroscopie donne la composition du plasma et le comportement $1 \mathrm{D}$ de chaque espèce détectée.

Avec la caméra rapide $\mathrm{CCD}$, pour différents délais à partir du tir laser, une photo du panache plasma est enregistrée avec des temps d'intégration de $25 \mathrm{~ns}$ à $100 \mu \mathrm{s}$ selon le gain choisi en fonction de la luminosité. Il est alors possible de suivre l'évolution du plasma sans distinction entre atomes neutres et ions.

Par spectroscopie optique d'émission, le plasma est analysé par tranches de $200 \mu \mathrm{m}$ en déplaçant perpendiculairement à la cible un sytème optique relié à un spectromètre. Ceci nous permet de suivre les espèces excitées (des ions jusqu'à deux fois ionisés et des neutres) en enregistrant leurs cinétiques. Un exemple typique de cinétiques est présenté figure 1 . L'évolution de leurs maxima en fonction du temps pour les différentes distances par rapport à la cible donne, une fois le fond continu intense soustrait, la vitesse la plus próbable de l'espèce observée $\left(10^{6} \mathrm{cms}^{-1}\right)$.

Dans le vide pour les deux méthodes, comme le décrivent R.K.Singh et J.Narayan[1], le panache plasma se propage linéairement. Les vitesses obtenues sont constantes et de l'ordre de $10^{6} \mathrm{cms}^{-1}$.

La propagation du plasma dans une ambiance gazeuse montre un comportement différent par rapport à celle du vide. La première différence est une durée de vie du plasma plus 
longue dans le gaz (jusqu'à $1 \mathrm{~ms}$ ) par rapport au vide (environ $3 \mu \mathrm{s}$ ). Ceci dénote bien une interaction entre matière vaporisée et gaz ambiant.

Généralement, dans une ambiance gazeuse, l'expansion du plasma est décrite par deux modèles : soit l'onde de choc, soit la force de viscosité [2,3]. Sur les photos, la formation d'une onde de choc semble apparaître dans la phase initiale de propagation du plasma selon une pression résiduelle propre à chaque gaz. Le modèle de l'onde de choc ne décrit pas systématiquement l'ensemble du comportement en fonction du temps pour tous les paramètres cible-gaz et pression du gaz [4]. Dans la phase suivante de propagation du plasma, le panache est freiné pour une distance d'arrêt propre à chaque cas. Ces deux modèles de l'hydrodynamique combinés ensemble s'accordent bien avec les résultats expérimentaux (figure 2). La modélisation complète de l'expansion semble néanmoins plus complexe.

Pour les premiers instants de l'expansion, la spectroscopie nous apporte un renseignement supplémentaire : le plasma se propage linéairement avec la même vitesse que celle dans le vide, et ce quelle que soit l'espèce (neutre ou ion). Les ions apparaissent en premier suivis par les neutres (recombinaison).

Ainsi, dans le gaz ambiant, le panache plasma se détache tout d'abord de la cible avec une expansion linéaire. Cette vapeur éjectée agit par la suite comme un piston sur le gaz, ce qui crée une onde de choc qui se propage à l'interface vapeur-gaz. Lorsque la pression à l'intérieur du panache plasma diminue et atteint celle du gaz ambiant, cette onde de choc disparait et le gaz commence alors à freiner le plasma jusqu'à le stopper.

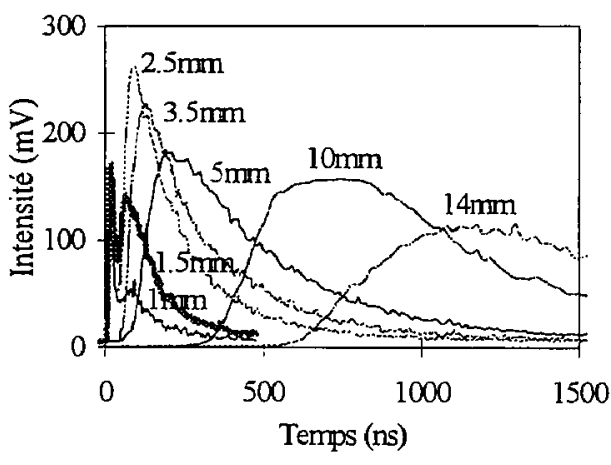

Figure 1: Cinétiques de la raie Hf II $3505 \AA$ dans $1 \mathrm{mb}$ d'Argon pour différentes distances par rapport à la cible.

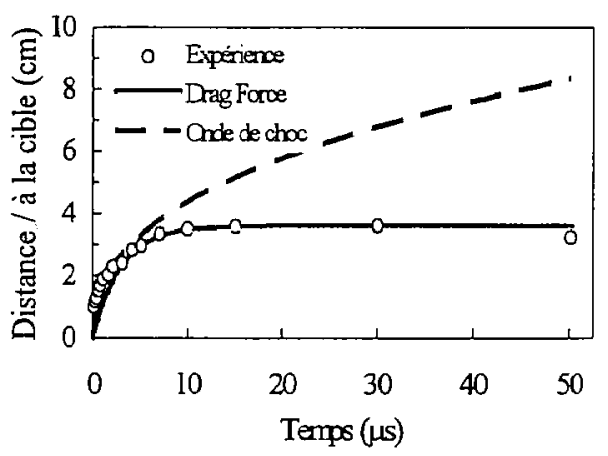

Figure 2: Modèles d'expansion pour le Hafrium dans $1 \mathrm{mb}$ d'Hélium.

\section{Références}

[1] Singh R.K., Narayan J., Phys. Rev. B 41 (13), (1990) 8843.

[2] Kools J.C.S., Jpn. J. Appl. Phys, 31 (1992) p.1964-1971.

[3] Geohegan D.B., Laser Ablation of Electronic Materials: Mechanisms and Applications, (E. Fogarassy et S. Lazare, North Holland, Amsterdam, 1992) p. 73-88.

[4] Stangl E., Anisimov S. et al, 'Dynamics of the vapor plume in laser materials ablation', Excimers lasers, Elounda (Crète, Grèce) 6-17 Sept 1993, L.D.Laude Eds,NATO ASI Series E, 265, p.79-90. 